\title{
Low Vacuum \& ESEM Imaging of Biological Specimens
}

\author{
D.J. Stokes
}

Polymers \& Colloids Group, University of Cambridge, Department of Physics, Cavendish Laboratory, Madingley Road, Cambridge, CB3 0HE, UK

Biological specimens can be imaged in low vacuum \& ESEM without preparation in the conventional sense. However, while the fixing, staining, drying and coating traditionally associated with other microscopic techniques is not usually required in low vacuum \& ESEM, the emphasis must necessarily shift to the treatment of specimens once inside the microscope chamber. Hence we need to consider how uncoated delicate biological specimens behave when subjected to electron irradiation in a partial vacuum, controlling and interpreting this as well as we are able, in order to obtain appropriate microstructural information.

For 'dry' specimens, such as polymeric \& porous materials, hard tissues (see Figure 1) and those with a tough or waxy exterior, the main criterion for low vacuum imaging is minimisation of charge build-up. Even so, there are a number of potential image interpretation issues involved, arising from selective localised specimen charging [1], positive ion behavior and electric fields in and around the specimen $[2,3]$.

For hydrated biological specimens, stability is a key issue - the specimen should be held in an atmosphere corresponding to its equilibrium state in order to prevent moisture loss or gain over time. Alternatively, pressures and temperatures can be chosen for non-equilibrium conditions, so that dynamic experiments involving evaporation or condensation of water can be carried out. Selection of suitable chamber pressures and specimen temperatures requires consideration of the phase diagram for water. However, it should be remembered that any aqueous phases in the specimen will contain dissolved solutes, which tend to lower the equilibrium vapor pressure needed, relative to pure water. Thus water vapor pressures corresponding to $50 \%-85 \%$ humidity, relative to pure water, are preferable [4]. Implicitly, imaging at lower pressures helps to reduce primary electron scattering, improving the quality of the results.

Uncoated specimens are sensitive to radiation damage, particularly at high magnifications and in a water vapor environment. A study involving polypropylene [5] showed that the deposition of condensed water layers on the polymer surface significantly increased the likelihood of damage: the highly mobile and reactive nature of radicals in liquid water increases the rate of polymer hydrolysis, compared to the dry state. Another study involving water vapor shows the influence of beam energy on the types and quantities of reactive species produced [6]. Care is therefore needed in order to avoid beam-induced artefacts, and a thorough assessment of a specimen's stability under the electron beam should always be carried out prior to engaging in experiments or interpreting results.

Furthermore, organic (low-Z) specimens possess low primary electron stopping powers, resulting in a large electron range within the specimen. This tends to decrease the spatial resolution relative to a coated specimen, where the coating helps to localise the electron signal at the specimen surface. Coatings also confer some protection against radiation damage, especially at high magnification. Hence we must choose objectively whether to coat or not, depending on what we wish to achieve. 


\section{References}

[1] D.J. Stokes et al., Scanning, 22(6) (2000) 357-365.

[2] J.P. Craven et al., J. Microscopy, 205(1) (2002) 96-105.

[3] M. Toth et al., J. Microscopy, 205(1) (2002) 86-95.

[4] D.J. Stokes et al., Scanning (2003, submitted).

[5] S. Kitching and A.M. Donald, J. Microscopy, 190 (1998) 357-365.

[6] C.P. Royall et al., J. Microscopy, 204(3) (2001) 185-195.

[7] This work was funded by a Royal Society Warren Research Fund Dorothy Hodgkin Research Fellowship.

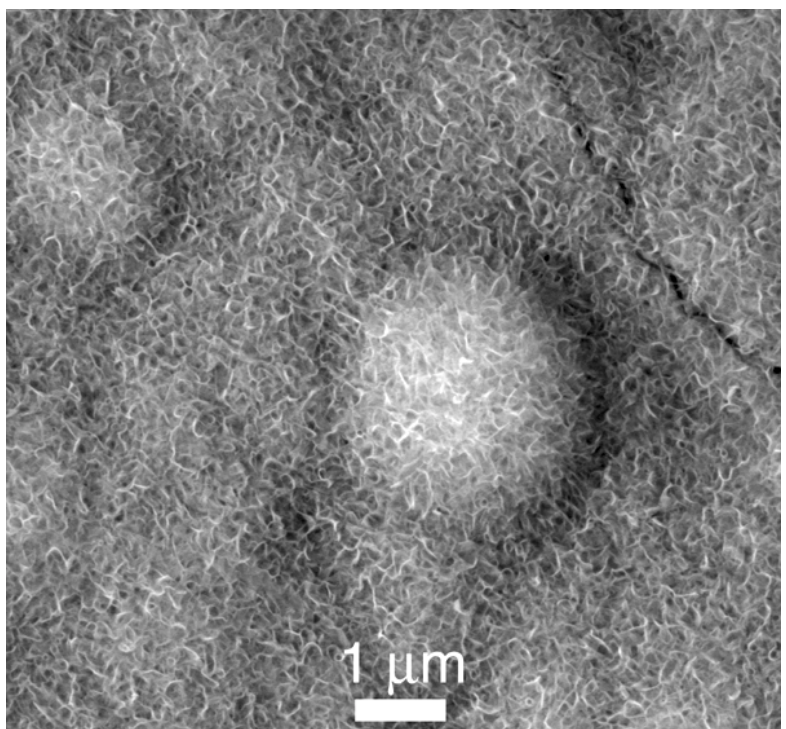

Figure 1. High magnification low vacuum image showing a physiological apatite layer (essential for bone formation) deposited on a biomaterial surface. Fine microstructure can be distinguished, having dimensions of approximately $200 \mathrm{~nm}$ x $10 \mathrm{~nm}$. Specimen courtesy of Claudia Bothelo.

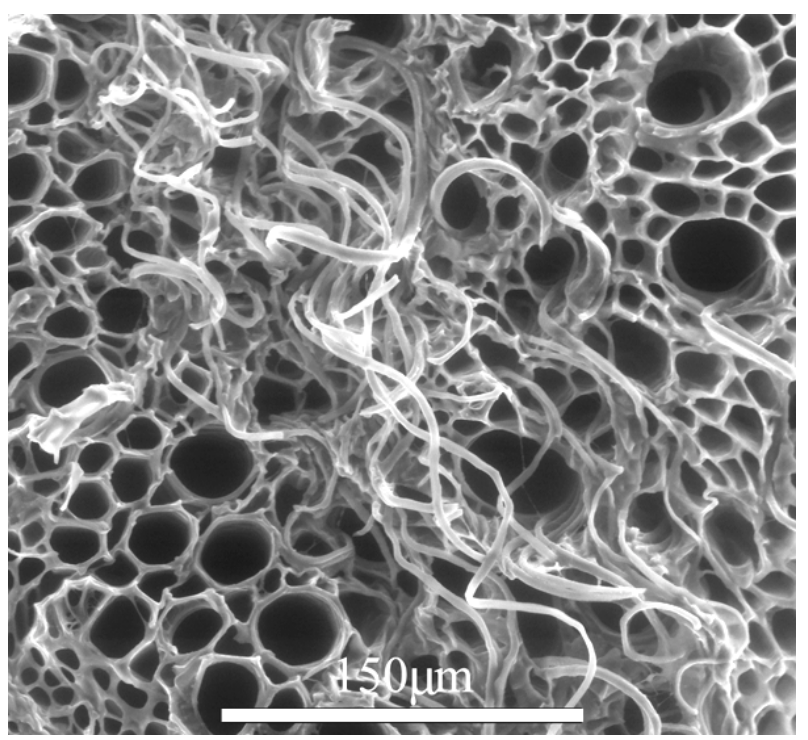

Figure 2. ESEM image of $\mathrm{x}$-section through vegetable tissue, showing cellular structure. 